\title{
Human emotion characterization by heart rate variability analysis guided by respiration
}

\author{
María Teresa Valderas ${ }^{1,3}$, Juan Bolea ${ }^{1,2}$, Michele Orini ${ }^{4}$, Pablo Laguna ${ }^{1,2}$, Fellow IEEE, Carlos Orrite ${ }^{5}$, \\ Montserrat Vallverdú ${ }^{2,3}$ and Raquel Bailón ${ }^{1,2}$
}

\begin{abstract}
Developing a tool which identifies emotions based on their effect on cardiac activity may have a potential impact on clinical practice, since it may help in the diagnosing of psychoneural illnesses. In this study, a method based on the analysis of heart rate variability (HRV) guided by respiration is proposed. The method was based on redefining the high frequency (HF) band, not only to be centered at the respiratory frequency, but also to have a bandwidth dependent on the respiratory spectrum. The method was first tested using simulated HRV signals, yielding the minimum estimation errors as compared to classic and respiratory frequency centered at $\mathrm{HF}$ band based definitions, independently of the values of the sympathovagal ratio. Then, the proposed method was applied to discriminate emotions in a database of video-induced elicitation. Five emotional states, relax, joy, fear, sadness and anger, were considered. The maximum correlation between HRV and respiration spectra discriminated joy vs. relax, joy vs. each negative valence emotion, and fear vs. sadness with $p$-value $\leq 0.05$ and $\mathrm{AUC} \geq 0.70$. Based on these results, human emotion characterization may be improved by adding respiratory information to $\mathrm{HRV}$ analysis.
\end{abstract}

Index Terms-Emotion recognition, autonomic nervous system, heart rate variability, respiration, spectral analysis, biomedical signal processing.

\section{INTRODUCTION}

Developing a tool which identifies human emotions may have a potential value in several fields. First, in the clinical practice, it may have value to reduce the diagnostic time of a psycho-neural illness, and, subsequently, it could directly represent a beneficial economic impact for the health system. Secondly, it can improve on the human-machine interaction since it could provide knowledge regarding the affective state of a user, bringing the machine closer to the human by including emotional content in the communication [1].

Several strategies have been proposed for emotion recognition in the area of non-invasive biosignals as electroencephalography (EEG) [1]-[6], galvanic skin response (GSR) [7], [8], skin temperature variation (ST), electrodermal activity [9] and electrocardiography (ECG) [10]-[13], among others.

\footnotetext{
${ }^{1}$ Biomedical Signal Interpretation and Computational Simulation (BSICoS), Aragón Institute for Engineering Research (I3A), IIS Aragón, University of Zaragoza, Spain, María de Luna, 1, 50015 Zaragoza, Spain.

${ }^{2}$ CIBER de Bioingeniería, Biomateriales y Nanomedicina (CIBER-BBN), Spain.

${ }^{3}$ Department ESAII, Centre for Biomedical Engineering Research, Universitat Politècnica de Catalunya, Barcelona, 08028, Spain.

${ }^{4}$ Institute of Cardiovascular SCHFence, University College of London, UK

${ }^{5} \mathrm{CV}$ Lab, Computer Vision Laboratory, I3A., University of Zaragoza, Spain, María de Luna, 1, 50015 Zaragoza, Spain.

Corresponding author at: Aragón Institute for Engineering Research (I3A), IIS Aragón, University of Zaragoza, 50018 Zaragoza, Spain. E-mail address: maite@maitevalderas.com (MT. Valderas).
}

This work has been focused on emotion recognition by means of heart rate variability (HRV) analysis.

Emotions activate biochemical mechanisms at the level of the hypothalamus, pituitary, and other peripheral glands. These tend to restore or suppress the immune and endocrine responses, making the development of diverse pathological processes possible [14]. Transient behaviour of the cardiovascular function is often linked with some emotional responses. In particular, heart rate is profoundly influenced by neural inputs from sympathetic and parasympathetic divisions of the autonomic nervous system (ANS), which allows the modification of cardiac function to meet the changing homeostatic needs of the body [15]. For example, cardiovascular reaction to a perceived stress situation creates an increase in blood pressure as a consequence of a general increase in cardiovascular sympathetic nerve activity and a decrease in parasympathetic activity [15]-[17]. When adrenergic sympathetic fibers activate, they release noradrenaline (NA) on cardiac cells, increasing the heart rate. When cholinergic parasympathic nerve fibers activate, they release acetylcholine on cardiac muscle cells and the heart rate decelerates [18]. Sympathetic and parasympathetic activation work to increase and decrease cardiac pumping, respectively [19]. Usually, an increment in parasympathetic nerve activity is accompanied by a reduction in sympathetic nerve activity, and vice versa.

In previous studies, recognition of emotional states assessed by means of HRV spectral analysis has been reported [11], [20]-[25]. HRV spectral analysis typically considers the power in three bands: a) very low frequency (VLF) component in the range between $0 \mathrm{~Hz}$ and $0.04 \mathrm{~Hz}$, b) low frequency (LF) component between $0.04 \mathrm{~Hz}$ and $0.15 \mathrm{~Hz}$, and c) high frequency (HF) component between $0.15 \mathrm{~Hz}$ and $0.40 \mathrm{~Hz}$ [26].

It is well known that HRV is influenced by respiration. Heart rate is increased during inspiration and reduced during expiration, phenomenon described as Respiratory Sinus Arrhythmia (RSA). RSA has been used as an index of cardiac vagal or parasympathetic function, usually measured by the HF component of the HRV [27], while the LF component is affected by both sympathetic and parasympathetic activity. The necessity of redefining the HF band to be centered on the respiratory frequency when respiratory frequency $\left(F_{R}\right)$ is above $0.40 \mathrm{~Hz}$, has already been highlighted, as well as the misinterpretation of spectral HRV indices when respiratory frequency lies within the LF band [28].

Several studies have already used respiratory information to define the HF band. Most of them define the HF band centered at respiratory frequency and use a fixed bandwidth. Only a few of them use variable HF bandwidth dependent 
on respiration. In [29], respiratory frequency as well as its rate of variation were used to estimate HF power based on a parametric decomposition of the instantaneous autocorrelation function. In [30], an HF bandwidth dependent on respiration stability was used to analyze HRV in critically ill patients. Recently, spectral coherence between respiration and HRV has been used to define the HF band [31], [32].

Moreover, the relationship between respiration and HRV might be further exploited to add relevant information regarding ANS regulation. Interactions between respiration and HRV have been continuously assessed using time-varying spectral coherence, partial coherence and phase differences during orthostatic test and under selective autonomic blockade [33], [34]. Characterization of these interactions might be crucial in applications where both respiration and HRV are altered, such as during stress [13].

In this work, we propose the joint analysis of HRV and respiration to improve human emotion characterization. HF band is defined based on the maximum spectral correlation between HRV and respiration. Both the center and bandwidth of HF band depend on respiration. The maximum spectral correlation itself is proposed as an index to identify emotions. Our hypothesis is that this index, characterizing the relationship between respiration and HRV, can add relevant information to HRV analysis to describe human emotions.

First, a simulation study is designed to evaluate the ability of the proposed HF band to quantify RSA. The performance of the proposed HF band is compared to other commonly used HF band definitions. Then, the ability of the proposed indices to characterize human emotions will be tested on a database of video-induced emotions.

\section{METHODS AND MATERIALS}

\section{A. Emotion database}

A database of 25 volunteers was recorded at the University of Zaragoza during an induced emotion experiment. It contains the simultaneous recording of ECG and respiration using a MP100 BIOPAC device. The limb ECG leads I, II and III were sampled at $1 \mathrm{kHz}$ and the respiration signal, $r(t)$, at 125 Hz. The distribution of male (12) and female (13) were: four men and five women in the age range [18-35] years, four men and four women in the age range [36-50] years and four men and four women over 50 years.

The following emotions were induced using videos: joy, fear, anger and sadness. Each subject was required to watch 8 different videos (two videos per emotion) in 2 days. The first day were recorded sessions 1 and 2, while sessions 3 and 4 were recorded in the second day. In session 1 and 4, the subject was stimulated with videos of joy and fear, and with videos of anger and sadness in session 2 and 3 . The videos of each session were presented in randomized order. Each video was preceded and followed by a relaxing video considered as baseline, to ensure that the physiological parameters returned to the baseline condition. A schema of the organization of the video-induced emotion sessions is represented in Fig. 1.

The contents of the videos were: the joy videos were excerpts from laughing monologues; the fear videos were

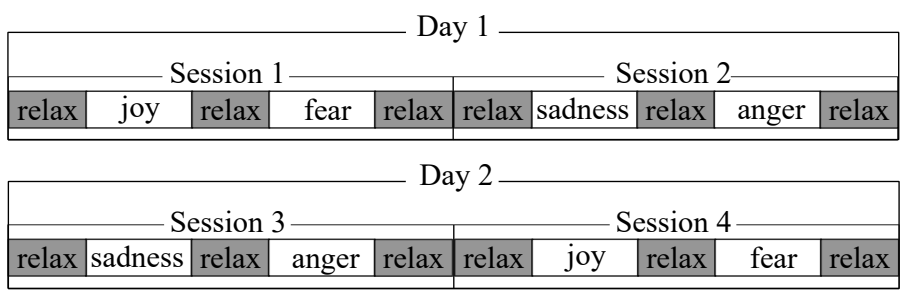

Fig. 1. Scheme of the organization of the video-induced emotion sessions. Session 1 and 2 were recorded the first day, and session 3 and 4 were recorded the second day. In session 1 and 4, the subject was stimulated with videos of joy and fear, and with videos of anger and sadness in session 2 and 3. All videos were presented in randomized order.

excerpts from scary movies, like Alien and Misery; the sadness videos were an excerpt from the film The Passion of the Christ and a documentary film about history wars; the anger videos were an excerpt of the documentary film of the Columbine High School massacre in 1999 and a documentary about domestic violence; and the relax videos were excerpts from nature images with classical music.

All videos were five minutes long, except one of the videos corresponding to emotion fear, which lasted three minutes. The Institution's Ethical Review Board approved all experimental procedures involving human subjects and the subjects gave their written consent.

The emotion database has been validated by 16 subjects, different from the ones participating in the database, using the Positive and Negative Affect Schedule - Expanded Form (PANAS-X) [35]. To assess specific emotional states, a 60-item scale is used. Based on the sum of specific items, the following affect scales can be computed: fear, sadness, guilt, hostility, shyness, fatigue, surprise, joviality, self-assurance, attentiveness and serenity. Then, a Basic Negative Emotion (BNE) scale is defined as the average of sadness, guilt, hostility and fear scales, and a Basic Positive Emotion (BPE) scale as the average of joviality, self-assurance and attentiveness scales. In this work we studied the BPE, BNE, joviality, fear, sadness and hostility scales.

\section{B. Signal Preprocessing}

Beat occurrence times were detected from the recorded ECG using a wavelet-based detector [36]. Instantaneous heart rate $\left(d_{H R}(t)\right)$ was estimated from the beat occurrence times based on the integral pulse frequency modulation (IPFM) model, which takes into account the presence of ectopic beats [37]. A time-varying mean heart rate $\left(d_{H R M}(t)\right)$ was computed by low pass filtering (cut-off frequency $0.03 \mathrm{~Hz}) d_{H R}(t)$, and then the HRV was obtained as $d_{H R V}(t)=d_{H R}(t)-d_{H R M}(t)$. The modulating signal, $m(t)$, which is assumed to carry the ANS information according to the IPFM model [38], was estimated as $m(t)=\left(d_{H R}(t)-d_{H R M}(t)\right) / \overline{d_{H R M}}$ [38], being $\overline{d_{H R M}}$ the mean of $d_{H R M}(t)$. The $m(t)$ was resampled at $4 \mathrm{~Hz}$.

The respiratory signal, $r(t)$, was filtered by a band pass filter from $0.04 \mathrm{~Hz}$ to $0.80 \mathrm{~Hz}$, which is assumed to cover the physiological frequency range for $m(t)$ and $r(t)$, and undersampled at $4 \mathrm{~Hz}$. 


$$
\rho_{(S m, S r)}^{a b}=\frac{\int_{a}^{b}\left(S_{m}(f)-\overline{S_{m}}(f)\right)\left(S_{r}(f)-\overline{S_{r}}(f)\right) d f}{\sqrt{\int_{a}^{b}\left(S_{m}(f)-\overline{S_{m}}(f)\right)^{2} d f \int_{a}^{b}\left(S_{r}(f)-\overline{S_{r}}(f)\right)^{2}} d f}
$$

Spectral HRV indices were estimated from the power spectrum density (PSD) of $m(t)\left(S_{m}(f)\right)$, computed by means of the Welch Periodogram. Then, the power content in the HF band $\left(P_{H F}\right)$ and in the LF band $\left(P_{L F}\right)$, the normalized power in the LF band (i.e. $\left.P_{L F n}=P_{L F} /\left(P_{L F}+P_{H F}\right)\right)$ and the ratio $R=P_{L F} / P_{H F}$ were computed. The limits of the bands are defined in Section II.C. The respiratory frequency $F_{R}$ was estimated from the location of the largest peak in the PSD obtained from $r(t)$ $\left(S_{r}(f)\right)$.

\section{Frequency band definition}

1) Shifted and resized HF band based on Spectrum Correlation $(S C H F)$ : The HF band is redefined based on the correlation between $S_{m}(f)$ and $S_{r}(f)$ as given in Eq. (1), where $a$ and $b$ are the lower and upper limits of the analyzed frequency range. The maximum value of $\rho_{(S m, S r)}^{a b}$ is searched, following the steps detailed below:

- Step 1: the spectral correlation of $S_{m}(f)$ and $S_{r}(f)$, $\rho_{(S m, S r)}^{a b}$, is computed within a bandwidth of $0.02 \mathrm{~Hz}$ centered at $F_{R}$.

- Step 2: the integration frequency range $[a, b]$ is symmetrically expanded $0.02 \mathrm{~Hz}$ and $\rho_{(S m, S r)}^{a b}$ is recomputed. This step is repeated until the physiological range from $0.1 \mathrm{~Hz}$ to $\overline{d_{H R M}} / 2$ is covered, with the following restrictions: (1) the lower limit $a$ must be above $0.10 \mathrm{~Hz}$, (2) the upper limit $b$ must be below half the mean heart rate $\left(\overline{d_{H R M}} / 2\right)$ and (3) $S_{r}(b)$ must be above $5 \%$ of the maximum value of $S_{r}(f)$ to avoid including in the correlation estimation frequencies with no respiratory power. In these cases, the restricted limit (lower or upper) is kept fixed and the other limit is increased in $0.01 \mathrm{~Hz}$. The resulting integration frequency ranges are no longer symmetric with respect to $F_{R}$.

- Step 3: the maximum value of $\rho_{(S m, S r)}^{a b}$, denoted by $\rho_{\max }=\rho_{(S m, S r)}^{a_{\max } b_{\max }}$, determines the lower and upper limits of the $\left[a_{\max }, b_{\max }\right]$ redefined $\mathrm{HF}$ band $\left(\mathrm{HF}_{S C}\right)$.

Only those recordings showing $\rho_{\max } \geq 0.5$ were considered for further analysis, being this value selected empirically as a trade-off between subject number inclusion and correlation strength. Fig. 2 shows a diagram of the SCHF method.

Standard LF band was considered in the range of [0.04, $0.15] \mathrm{Hz}$, except when the HF band encroached the LF band. In these cases, the upper limit of the LF band was reduced to the lower limit of the HF band, i.e., LF band was $\in[0.04$, $\left.a_{\max }\right] \mathrm{Hz}$.

2) Classic HF band: The classic HF band described in Task Force [26] was analyzed, i.e. [0.15, 0.40] Hz.

3) Shifted $H F$ band centered at $F_{R}$ with fixed bandwidth: As defined in previous studies [28], [39], the HF band was centered at $F_{R}$ and had a fixed bandwidth of $0.11 \mathrm{~Hz}\left(H F_{F_{R}}\right)$.

In approaches to $H F_{S C}$ and $H F_{F_{R}}$, which take into account respiratory information, those recordings with $F_{R}<0.1 \mathrm{~Hz}$ are

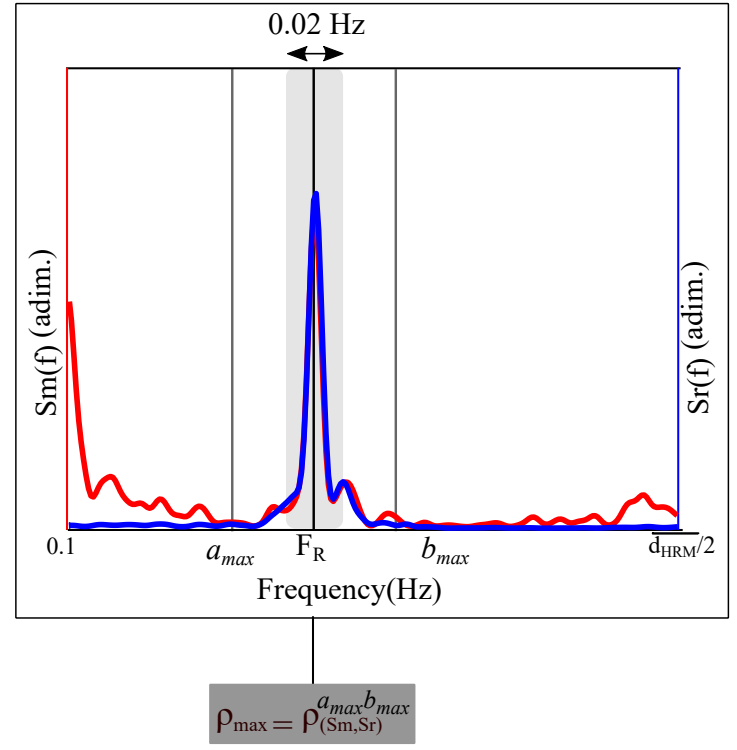

Fig. 2. Diagram of the SCHF methodology: PSD of $m(t)\left(S_{m}(f)\right)$ and PSD of $r(t)\left(S_{r}(f)\right)$. The correlation between $S_{m}(f)$ and $S_{r}(f)$ was calculated by expanding symmetrically the $[a, b]$ range in steps of $0.02 \mathrm{~Hz}$ per iteration. The maximum value of the correlation between $S_{m}(f)$ and $S_{r}(f)\left(\rho_{\max }\right)$ determines the lower and upper limits $\left(a_{\max }, b_{\max }\right)$ of the redefined $\mathrm{HF}$ band $\left(\mathrm{HF}_{S C}\right)$.

excluded from the analysis due to the overlapping between the LF and HF bands.

\section{Simulation study}

A simulation study was carried out to validate the proposed $H F_{S C}$ definition.

Synthetic modulating signals $\left(m_{s}(t)\right)$ were generated as the sum of a HF and a LF component, following the steps detailed below:

- Step 1: the HF component was obtained by filtering a respiration signal $r(t)$ from the emotion database from $0.25 \mathrm{~Hz}$ to $\overline{d_{H R M}} / 2$. This HF component is denoted by $m_{H F_{i}}(t), \mathrm{i}=1, \ldots, \mathrm{I}$, where $\mathrm{I}$ is the number of cases with $F_{R}>0.35 \mathrm{~Hz}$ since those are the most challenging for the classic HF band. A total of $\mathrm{I}=59$ cases were identified.

- Step 2: the LF component was simulated based on a timevarying autoregressive moving average (ARMA) model [40]. The frequency for the ARMA model was obtained as the maximum of the original modulating signal spectrum $S_{m}(f)$, associated with the i-th subject, in the band from $0.04 \mathrm{~Hz}$ to $0.15 \mathrm{~Hz}$ and the amplitude was fixed to 0.1 . A total of 50 realizations of the LF component were generated for each considered subject, yielding $m_{L F_{i}}{ }^{k}(t)$ with $\mathrm{k}=1, \ldots, 50$.

- Step 3: the simulated modulating signals were constructed as $m_{s_{i}}{ }^{k}(t)=m_{L F_{i}}{ }^{k}(t)+\alpha m_{H F_{i}}(t)$, where the $\alpha$ parameter allows to simulate a set of sympathovagal ratios, $R$. The following $R$ were considered: $0.5,1,2,5,10$, 


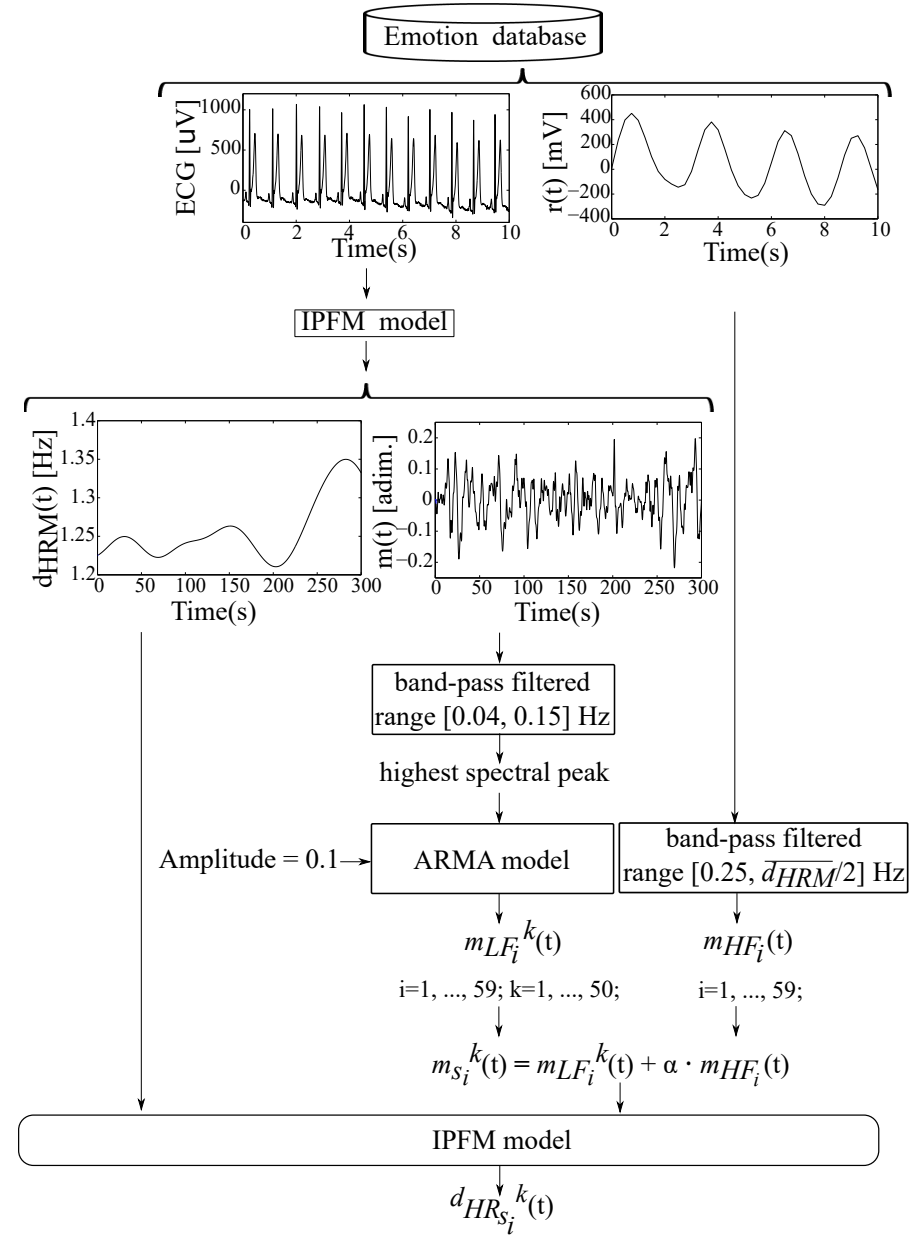

Fig. 4. Schema of the simulation process for a single recording detailed in the following steps: (1) the HF component of the synthetic $m(t)$ signals was obtained by filtering the $r(t)$ of the emotion database from $0.25 \mathrm{~Hz}$ to the $\overline{d_{H R M}} / 2$, resulting in $m_{H F_{i}}(t)$, (2) the LF component was simulated by an ARMA model with a fixed amplitude of 0.1 and a frequency calculated by the maximum of the original $S_{m}(f)$, associated with the $i$-th subject, resulting in $m_{L F_{i}}{ }^{k}(t)$, (3) the simulated modulating signals $m_{s_{i}}{ }^{k}(t)$ were constructed as the sum of the LF and HF components, where $i$ is the number of the subject analyzed and $k$ the number of the realization performed and (4) each modulating signal $m_{s_{i}}{ }^{k}(t)$ fed an IPFM model with time-varying threshold $\left(1 / d_{H_{R M} M_{i}}(t)\right)$ which generates the beat occurrence times, and from them the HRV signal $d_{H R_{s_{i}}}{ }^{k}(t)$ is derived.

15, 20 and 30, as shown in Fig. 3. This range allows to cover the physiological $R$ values computed during pure parasympathetic stimulation, median (interquartile range) of $1.53(0.83 \mid 2.11)$ and pure sympathetic stimulation $19.52(11.80 \mid 27.75)$ in a database of healthy subjects during pharmacological blockade and body position changes [41].

- Step 4: finally, each modulating signal $m_{s_{i}}{ }^{k}(t)$ fed an IPFM model with time-varying threshold which generates the beat occurrence time series [38]. The time-varying threshold is defined as $1 / d_{H R M_{i}}(t)$. From the simulated beat occurrence time series, a simulated instantaneous heart rate was obtained $d_{H R_{S_{i}}}{ }^{k}(t)$. The same processing described in section II.B for real signals was applied to simulated $d_{H R_{S_{i}}}{ }^{k}(t)$. A diagram of the whole process is shown in Fig. 4.

\section{E. Performance measurement}

The mean relative error (MRE) of HF power was calculated for each ratio Eq. (2).

$$
\operatorname{MRE}(\%)=\text { mean }\left(\frac{P_{H F_{i}}{ }^{k}-P_{H F r_{i}}}{P_{H F r_{i}}}\right) 100
$$

Where $P_{H F_{i}}{ }^{k}$ was the spectral content in the HF band, calculated as explained in Section II.C, from the simulated

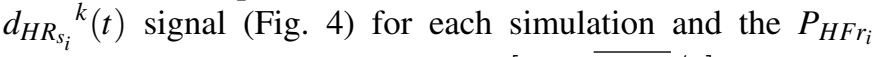
was the reference spectral content $\in\left[0.25, \overline{d_{H R M}} / 2\right] \mathrm{Hz}$ derived from $d_{H R_{s_{i}}}{ }^{k}(t)$ signal.

The proposed SCHF methodology was compared with the other HF band definitions. Therefore, $P_{H F_{i}}{ }^{k}$ and $P_{H F r_{i}}$ for the MRE calculation were computed according to the bandwidth definitions detailed in Section II.C: (1) $\mathrm{HF}_{S C}$, (2) HF and (3) $\mathrm{HF}_{F_{R}}$.

\section{F. Statistical analysis}

Prior to the statistical analysis, normality distribution of all indices was evaluated by Lillie test.

Statistical analysis was done by T-test or Wilcoxon-test when necessary, depending on normality test results to evaluate differences for all followed paired conditions: relax vs. joy (R$\mathrm{J}$ ), relax vs. fear (R-F), relax vs. sadness (R-S), relax vs. anger (R-A), joy vs. fear (J-F), joy vs. sadness (J-S), joy vs. anger (J-A), fear vs. sadness (F-S), fear vs. anger (F-A) and sadness vs. anger (S-A).

Firstly, the affect scales BPE, BNE, joviality, fear, sadness and hostility have been statistically evaluated for database validation. Subsequently, the following HRV indices have been analyzed:

- Indices derived from the $\mathrm{HF}_{S C}$ band: $P_{H F_{S C}}, P_{L F n_{S C}}, R_{S C}$, $\triangle H F, a_{\max }$ and $b_{\max }$ and the novel index proposed in this work $\rho_{\max }$. The respiratory frequency of the recordings which accomplishes all the restrictions imposed in Section II.C, denoted by $F_{R_{S C}}$ was also considered.

- Indices derived from the classic HF band: $P_{H F}, P_{L F n}$ and $R$. The respiratory frequency, $F_{R}$, of all recordings was also studied.

- Indices derived from the $\mathrm{HF}_{F_{R}}$ band: $P_{H F_{F_{R}}}, P_{L F n_{F_{R}}}$ and $R_{F_{R}}$. The respiratory frequency of the recordings which accomplishes the unique restriction of $F_{R} \geq 0.10 \mathrm{~Hz}$, denoted by $F_{R_{F_{R}}}$ was also considered.

The significant statistical level was $p$-value $\leq 0.05$, that provides a reliable value for statistical discrimination [42]. To analyze the capability of the indices to discriminate emotions, the area under the receiver operating characteristic curve (AUC) was calculated and only those indices with AUC $\geq 0.70$ were further considered. Finally, sensitivity, specificity and accuracy for each index in 2-class emotion classification were calculated using the leave-one-out cross validation method [43].

\section{RESULTS}

\section{A. Validation of the emotion database}

The validation of the emotion database was performed by mean of the PANAS-X scale [35]. 

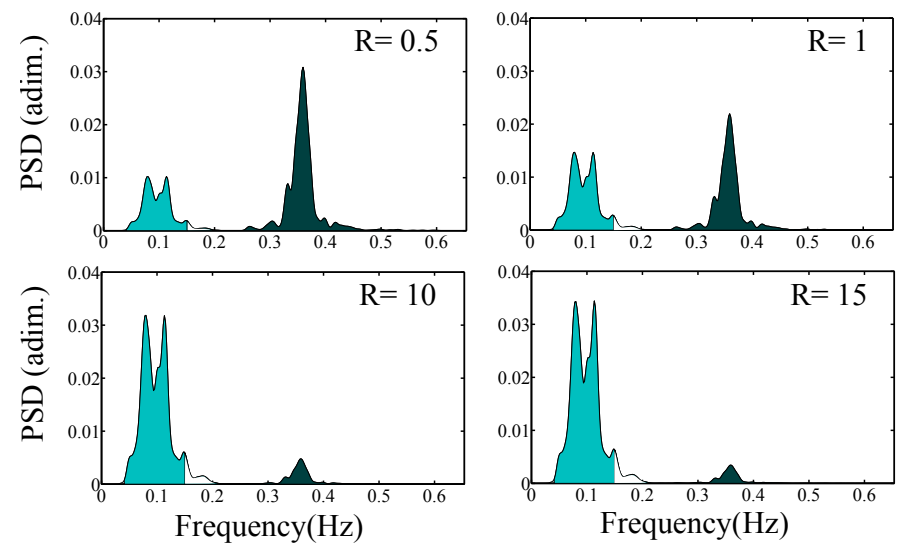

LF Band
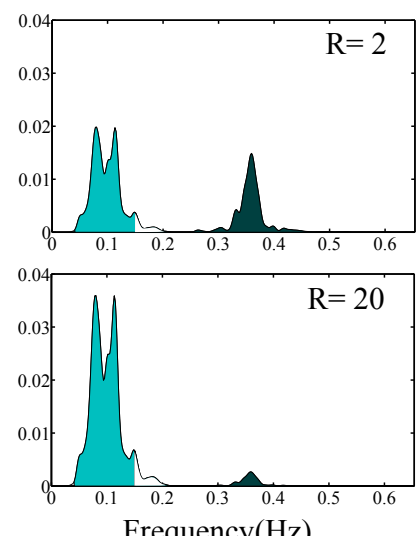

Frequency $(\mathrm{Hz})$

HF Band
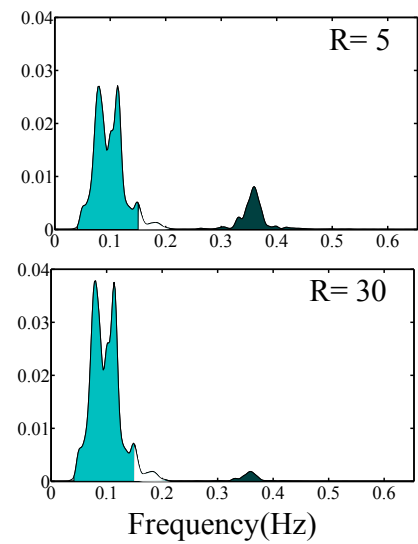

Frequency $(\mathrm{Hz})$

Fig. 3. PSD of the modulating signal simulated $d_{H R_{s_{i}}}{ }^{k}(t)$ for the physiological sympathovagal ratios: $0.5,1,2,5,10,15,20$ and 30.

TABLE I

MEAN AND STANDARD DEVIATION ( $M \pm S T D$ ) OF THE PANAS-X SCALES: Basic Positive Emotion (BPE), Basic Negative Emotion (BNE), JOVIALITY, FEAR, SADNESS AND HOSTILITY.

\begin{tabular}{|l|r|r|r|r|}
\cline { 2 - 5 } \multicolumn{1}{c|}{} & \multicolumn{4}{c|}{ Emotions } \\
\hline Scales & Joy & Fear & Sadness & Anger \\
\hline BPE & $13.1 \pm 4.8$ & $8.1 \pm 1.7$ & $7.4 \pm 1.5$ & $7.8 \pm 2.2$ \\
\hline BNE & $6.5 \pm 0.8$ & $12.2 \pm 3.9$ & $14.3 \pm 4.4$ & $12.8 \pm 3.8$ \\
\hline Joviality & $22.3 \pm 8.6$ & $9.0 \pm 2.4$ & $8.4 \pm 0.8$ & $8.8 \pm 1.6$ \\
\hline Fear & $6.4 \pm 1.1$ & $19.0 \pm 6.6$ & $14.8 \pm 5.3$ & $13.8 \pm 5.9$ \\
\hline Sadness & $5.2 \pm 0.6$ & $8.7 \pm 4.6$ & $14.8 \pm 5.5$ & $11.7 \pm 4.5$ \\
\hline Hostility & $8.1 \pm 1.7$ & $14.3 \pm 5.3$ & $15.7 \pm 5.4$ & $15.8 \pm 4.8$ \\
\hline
\end{tabular}

In Table I, the mean and standard deviation (M \pm STD) of the scales evaluated for each emotion are shown. It could be observed that the highest mean value of BPE scale corresponds to the emotion with positive valence (joy), while mean value of BNE was higher for emotions with negative valence (fear, sadness, anger). In addition, the affect scale with highest value in joy is joviality and the highest value in fear emotion is fear scale. However, there is not a single affect scale for sadness and anger that defines each emotion, resulting in high values for the fear, sadness and hostility scales.

Table II displays the $p$-values obtained in the comparison of PANAS-X scales between different emotions. All affect scales showed statistically significant differences between positive valence and negative valence emotions (J-F, J-S, J-A). Affect scales showing largest statistically significant differences between negative valence emotions were: fear scale (F-S, F-A) and sadness scale (F-S, S-A).

\section{B. Evaluation of the methods for synthetic data}

Fig. 5 presents the Mean and STD of the relative errors in PHF estimation obtained from $\mathrm{HF}_{S C}, \mathrm{HF}$ and $\mathrm{HF}_{F_{R}}$ for several physiological sympathovagal ratios, $R$ i.e. $0.5,1,2,5,10,15$, 20 and 30. The standard HF bandwidth presents relative error values strongly dependent on the ratio, while the $\mathrm{HF}_{F_{R}}$ and the $\mathrm{HF}_{S C}$ bandwidth presents lower relative error values regardless

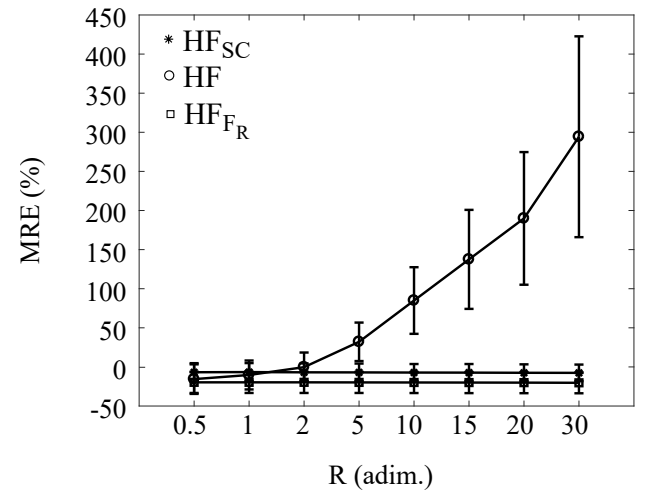

Fig. 5. Mean and standard deviation $(\mathrm{M} \pm \mathrm{STD})$ of the mean relative errors (MRE) obtained by Eq. (2) for $\mathrm{HF}_{S C}, \mathrm{HF}$ and $\mathrm{HF}_{F_{R}}$ methods for eight physiological sympathovagal ratios studied: $0.5,1,2,5,10,15,20$ and 30.

of the ratio values. Furthermore, the $\mathrm{HF}_{S C}$ bandwidth presents lower relative errors than the $\mathrm{HF}_{F_{R}}$ one.

\section{Evaluation of the methods for real data}

All indices derived from the $H F_{S C}, \mathrm{HF}$ and $H F_{F_{R}}$ bands have been evaluated and compared between each pair of emotions, however, only those parameters that revealed statistical differences to discriminate between pairs of emotions are shown.

In Fig. 6, the boxplots are shown in terms of median and interquartile ranges as first (Q1) and third (Q3) quartile, median (Q1|Q3) of: (a) $P_{L F n_{S C}}$, (b) $P_{L F n}$, (c) $P_{L F n_{F_{R}}}$, (d) $R_{S C}$, (e) $R$, (f) $R_{F_{R}}$ and (g) $\rho_{\text {max }}$ for the emotions studied.

The spectral indices $P_{L F n}$ and $R$ revealed statistically significant differences between R-J, J-F and J-S. However, $P_{L F n_{F_{R}}}$, $P_{L F n_{S C}}, R_{F_{R}}$ and $R_{S C}$ only show statistically significant differences between R-J and J-F. Additionally, the novel $\rho_{\max }$ provided statistically significant differences between R-J, JF, J-S, J-A and F-S. Since $\rho_{\max }$ obtained AUC $\geq 0.8$, its discrimination capability was further analyzed, calculating sensitivity, specificity and accuracy using cross validation (Table III).

Therefore, among all the emotions compared, neutral state vs. positive valence, positive valence vs. all negative valences 
TABLE II

$p$-VALUES OF THE PANAS-X SCALES: BASIC POSITIVE EMOTION (BPE), BASIC NEGATIVE EMOTION (BNE), JOVIALITY, FEAR, SADNESS AND HOSTILITY FOR THE PAIR OF EMOTIONAL CONDITIONS INDUCED BY VIDEOS: JOY VS. FEAR (J-F), JOY VS. SADNESS (J-S), JOY VS. ANGER (J-A), FEAR VS. SADNESS (F-S), FEAR VS. ANGER (F-A) AND SADNESS VS. ANGER (S-A).

\begin{tabular}{|l|c|c|c|c|c|c|}
\cline { 2 - 7 } \multicolumn{1}{c|}{} & \multicolumn{7}{c|}{ Emotions analyzed } \\
\hline Scales & $\mathrm{J}-\mathrm{F}$ & $\mathrm{J}-\mathrm{S}$ & $\mathrm{J}-\mathrm{A}$ & $\mathrm{F}-\mathrm{S}$ & $\mathrm{F}-\mathrm{A}$ & $\mathrm{S}-\mathrm{A}$ \\
\hline BPE & $p \leq 0.001$ & $p \leq 0.001$ & $p \leq 0.001$ & 0.011 & 0.043 & n.s. \\
\hline BNE & $p \leq 0.001$ & $p \leq 0.001$ & $p \leq 0.001$ & 0.005 & n.s. & 0.007 \\
\hline Joviality & $p \leq 0.001$ & $p \leq 0.001$ & $p \leq 0.001$ & n.s. & n.s. & n.s. \\
\hline Fear & $p \leq 0.001$ & $p \leq 0.001$ & $p \leq 0.001$ & $p \leq 0.001$ & $p \leq 0.001$ & n.s. \\
\hline Sadness & $p \leq 0.001$ & $p \leq 0.001$ & $p \leq 0.001$ & $p \leq 0.001$ & 0.002 & $p \leq 0.001$ \\
\hline Hostility & $p \leq 0.001$ & $p \leq 0.001$ & $p \leq 0.001$ & n.s. & n.s. & n.s. \\
\hline
\end{tabular}

TABLE III

SENSITIVITY, SPECIFICITY AND ACCURACY CALCULATED USING CROSS VALIDATION FOR THE PARAMETER $\rho_{\max }$ WITH AUC $\geq 0.8$ : RELAX VS. JOY (R-J), JOY VS. SADNESS (J-S) AND JOY VS. ANGER (J-A).

\begin{tabular}{|c|c|c|c|}
\cline { 2 - 4 } \multicolumn{1}{c|}{} & R-J & J-S & J-A \\
\hline Sensitivity (\%) & 66.7 & 88.9 & 99.9 \\
\hline Specificity (\%) & 91.7 & 66.7 & 63.6 \\
\hline Accuracy (\%) & 79.2 & 77.8 & 77.3 \\
\hline
\end{tabular}

and F-S were significantly different. No statistical differences were found in the comparison between neutral state vs. negative valences and anger vs. negative valences.

In Table IV, the median $(\mathrm{Q} 1 \mid \mathrm{Q} 3)$ for $F_{R_{S C}}, F_{R}$ and $F_{R_{F_{R}}}$ are shown. In the same Table IV, the indices $\Delta H F, a_{\max }$ and $b_{\max }$ derived from the SCHF methodology are shown. No statistical significant differences have been obtained for these indices.

Fig. 7 displays two examples where the SCHF method is especially useful:

(a) The $F_{R}$ is below $0.15 \mathrm{~Hz}$ and therefore the $\mathrm{HF}_{S C}$ band encroaches the classic LF band. In this particular case the $\mathrm{HF}_{S C}$ band limits are: $a_{\max }=0.10 \mathrm{~Hz}$ and $b_{\max }=0.29 \mathrm{~Hz}$. The $\mathrm{LF}_{S C}$ band is redefined from $0.04 \mathrm{~Hz}$ to $0.10 \mathrm{~Hz}$.

(b) $F_{R}$ is $0.40 \mathrm{~Hz}$ and the $\mathrm{HF}_{S C}$ upper band limit should be shifted to the right to consider all the RSA information. In this particular case, the $\mathrm{HF}_{S C}$ band limits are: $a_{\max }=$ $0.34 \mathrm{~Hz}$ and $b_{\max }=0.46 \mathrm{~Hz}$.

\section{DISCUSSION}

In this study four out of the six emotions defined by Ekman [44] were studied. Disgust and surprise were not considered and should be evaluated in a future study, although recent research supports the theory of four basic emotions instead of six [45].

All subjects in this experiment reported an agreement between the theoretical positive valence of joy elicitation and the emotion felt, and fear, sadness and anger were identified as negative emotions. According to the analysis of the affect scales derived from the PANAS-X scale, shown in Table I, it could be stated that: joy emotion presents the highest values for the BPE and joviality scales; all negative emotions presented lower BPE and higher BNE, as expected; fear emotion obtains the highest mean value for the affect scale fear; however, sadness and anger emotions have a high mean value for fear, sadness and hostility scales. As shown in Table II, all
PANAS-X affect scales were significantly different between joy and all negative valence emotions (fear, sadness, anger). Statistical differences between negative valence emotions were only found in a subset of PANAS-X affect scales, challenging their discrimination through HRV (Table II).

According to the simulation results, the SCHF method presented the lowest relative error values for HF content estimation independently of the considered low-to-high frequency ratio, $R$, values (Fig. 5). In this way, the choice of adaptive HF frequency limits may avoid physiological misinterpretations of HF power content, because frequency limits depend strongly on age and physiological conditions [46].

The statistical analysis presented in Fig. 6 revealed statistically significant differences between: (1) R-J, (2) J-F, (3) J-S, (4) J-A and (5) F-S with different parameters.

The SCHF methodology proposed in this study differentiated R-J, J-F, J-S, J-A and F-S by means of $\rho_{\max }$. No statistical differences were found for neutral state vs. negative valences and anger vs. negative valences.

Classic frequency indices $P_{L F n}$ and $R$ were able to discriminate between R-J, J-F and J-S. Note that emotions J-A and F-S were only distinguished by parameter $\rho_{\max }$ derived from the new method SCHF, which offered additional statistical significant information based on the relationship between HRV and respiration.

Regarding respiratory information, neither $F_{R}$, nor $F_{R_{F_{R}}}$, nor $F_{R_{S C}}$ showed statistical significant differences between all pairs of emotions studied. Hernando et al. [13] did not found significant differences in respiratory frequency between relax and stress. The $F_{R}$ index, computed from all recordings, showed a median value around $0.30 \mathrm{~Hz}$ and with a first quartile above $0.15 \mathrm{~Hz}$ for for relax, fear, sadness and anger. Therefore, in all these cases, the redefined $\mathrm{HF}$ band $H F_{S C}$ does not encroach the classic LF band. However, in the case of joy, $F_{R}$ presented the lowest median value of $0.18 \mathrm{~Hz}$ with a first quartile of $0.08 \mathrm{~Hz}$. For this reason and during joy elicitation, the $H F_{S C}$ could encroach the classic LF band. Therefore, this highlights the need to redefine the HF band, especially in joy condition.

For this reason, all cases presenting a $F_{R}$ inside the classic LF band, a redefinition of the HF classic band could improve the measurement of the HF band, as shown in Fig. 7 (a). A similar situation occurs in Fig. 7 (b) when $F_{R}$ is near to or above the classic upper limit of the HF band $(0.40$ $\mathrm{Hz}$ ), where the classic range $[0.15,0.40] \mathrm{Hz}$ could miss the 
(a)

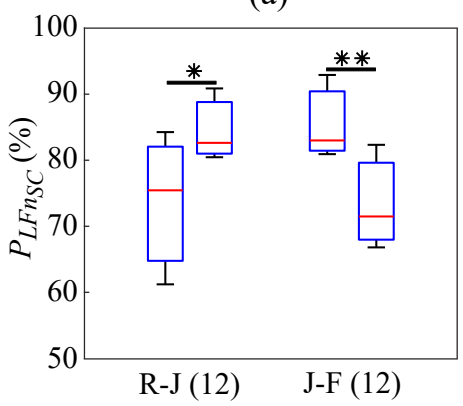

(d)

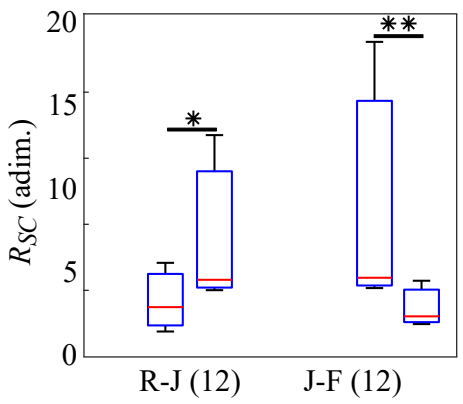

(b)

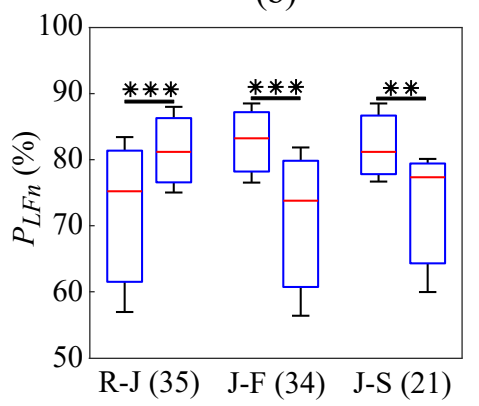

(e)

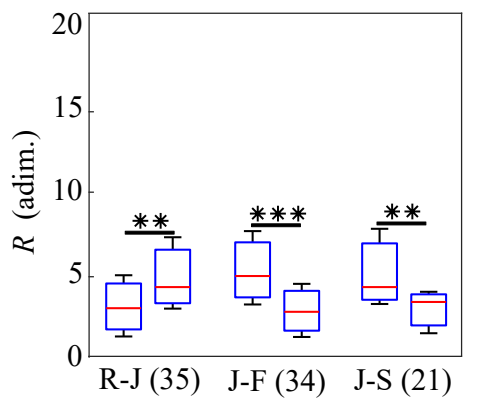

(c)

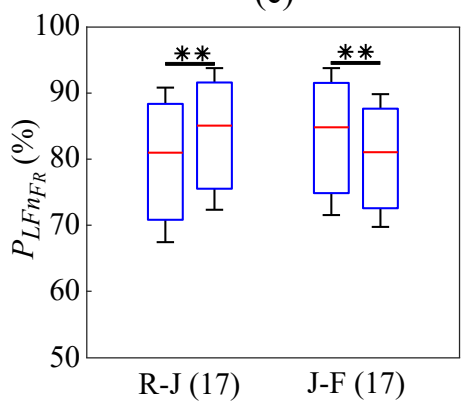

(f)

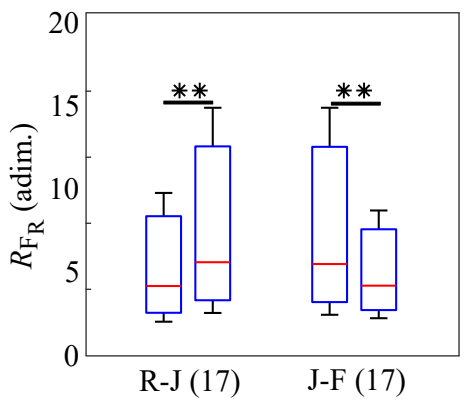

(g)

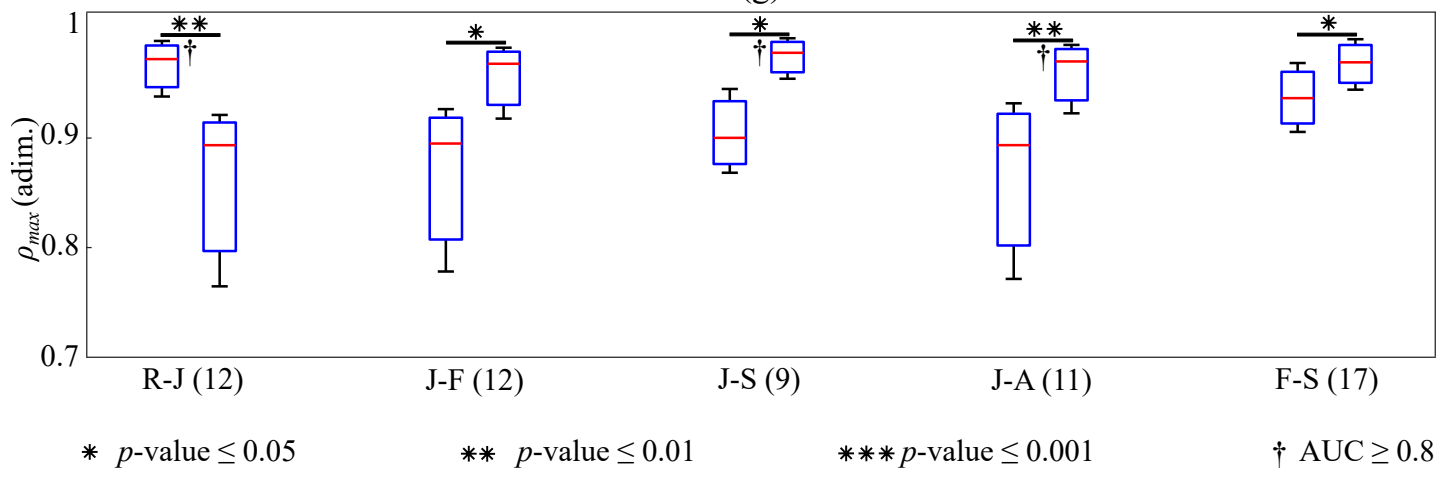

Fig. 6. Boxplots of the median (Q1|Q3) values of only those parameters which present statistical differences between the emotional conditions induced by videos: (a) $P_{L F n_{S C}}$, (b) $P_{L F n}$, (c) $P_{L F n_{F_{R}}}$, (d) $R_{S C}$, (e) $R$, (f) $R_{F_{R}}$ and (g) $\rho_{\max }$. The nomenclature used for each pair of emotions is: relax vs. joy (R-J), joy vs. fear (J-F), joy vs. sadness (J-S), joy vs. anger (J-A) and fear vs. sadness (F-S). The statistical differences between the pair of emotions are indicated by $*$ for $p$-value $\leq 0.05, * *$ for $p$-value $\leq 0.01, * * *$ for $p$-value $\leq 0.001$ and $\dagger$ for AUC $\geq 0.80$. Note that those indices which are not marked by a $\dagger$ have an AUC $\geq 0.70$. Together to the label of the pair of studied emotions in parentheses, it is the number of comparisons.
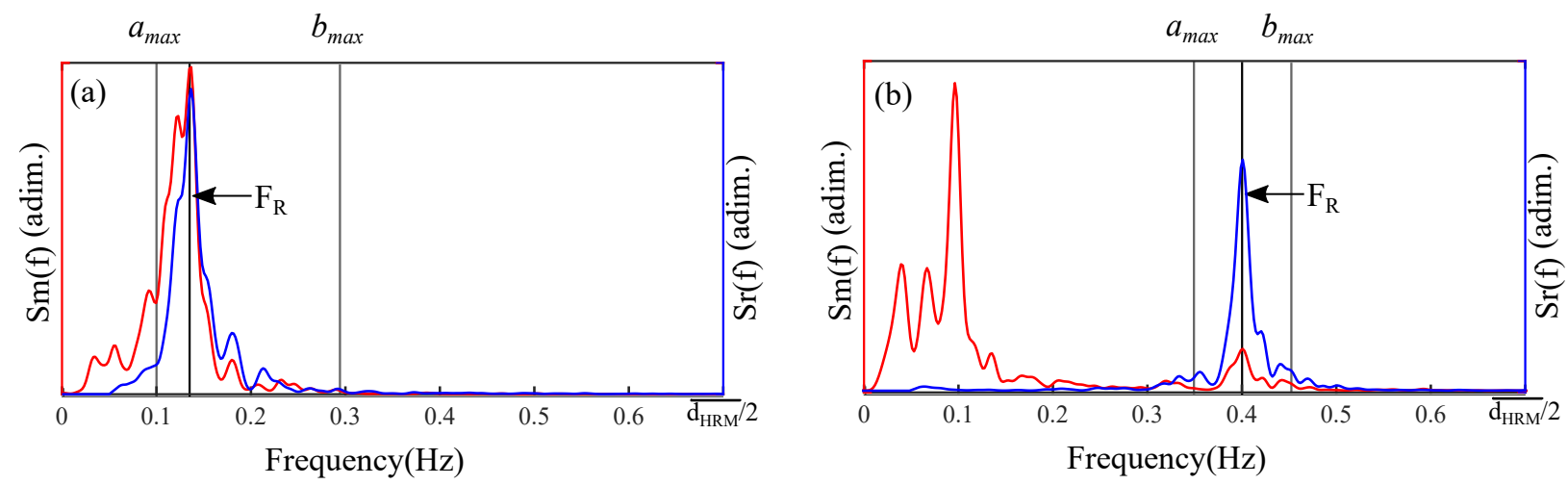

Fig. 7. Correlation between $S_{m}(f)$ and $S_{r}(f)$ in two particular cases: (a) $F_{R}$ is below $0.15 \mathrm{~Hz}$ and (b) $F_{R}$ is $0.40 \mathrm{~Hz}$.

RSA information. With a redefinition of the HF band in these cases, a more refined description of the physiological information could be extracted from the signals. However, only recordings which accomplished the restrictions of the SCHF method could be analyzed. This implies to discard an amount of signals from the analysis, and subsequently the number 
TABLE IV

MEDIAN (Q1|Q3) VALUES FOR $\Delta H F, a_{\max }, b_{\max }, F_{R_{S C}}, F_{R}$ AND $F_{R_{F_{R}}}$ FOR THE EMOTIONAL STATES STUDIED: STUDIED FOR RELAX, JOY, FEAR, SADNESS AND ANGER.

\begin{tabular}{|c|c|c|c|c|c|}
\cline { 2 - 6 } \multicolumn{1}{c|}{} & Relax & Joy & Fear & Sadness & Anger \\
\hline$\Delta H F(\mathrm{~Hz})$ & $0.16(0.12 \mid 0.20)$ & $0.18(0.14 \mid 0.23)$ & $0.14(0.12 \mid 0.18)$ & $0.16(0.12 \mid 0.18)$ & $0.14(0.12 \mid 0.18)$ \\
\hline$a_{\max }(\mathrm{Hz})$ & $0.21(0.18 \mid 0.27)$ & $0.25(0.22 \mid 0.27)$ & $0.24(0.20 \mid 0.26)$ & $0.24(0.20 \mid 0.27)$ & $0.24(0.21 \mid 0.27)$ \\
\hline$b_{\max }(\mathrm{Hz})$ & $0.40(0.35 \mid 0.42)$ & $0.41(0.39 \mid 0.51)$ & $0.40(0.36 \mid 0.44)$ & $0.40(0.36 \mid 0.45)$ & $0.40(0.36 \mid 0.43)$ \\
\hline$F_{R_{S C}}(\mathrm{~Hz})$ & $0.30(0.27 \mid 0.35)$ & $0.33(0.31 \mid 0.38)$ & $0.32(0.29 \mid 0.35)$ & $0.32(0.28 \mid 0.36)$ & $0.32(0.29 \mid 0.36)$ \\
\hline$F_{R}(\mathrm{~Hz})$ & $0.29(0.24 \mid 0.35)$ & $0.18(0.08 \mid 0.33)$ & $0.31(0.27 \mid 0.35)$ & $0.31(0.28 \mid 0.36)$ & $0.30(0.25 \mid 0.35)$ \\
\hline$F_{R_{F_{R}}}(\mathrm{~Hz})$ & $0.30(0.27 \mid 0.35)$ & $0.32(0.15 \mid 0.35)$ & $0.32(0.28 \mid 0.35)$ & $0.32(0.28 \mid 0.36)$ & $0.31(0.27 \mid 0.35)$ \\
\hline
\end{tabular}

of analyzed subjects in each case is reduced. Note that the percentage of subjects excluded is different for each of the comparisons, with a minimum of $22.7 \%$ for the comparison S-A and a maximum of $65.7 \%$ for the comparison R-J.

Classic $\Delta H F[0.15,0.40] \mathrm{Hz}$ has a bandwidth of $0.25 \mathrm{~Hz}$. Analyzing the results obtained by the SCHF method, the $\Delta H F$ presented a median bandwidth value of $0.16 \mathrm{~Hz}$ for relax, $0.18 \mathrm{~Hz}$ for joy, $0.14 \mathrm{~Hz}$ for fear and anger and $0.16 \mathrm{~Hz}$ for sadness. The lower and upper limit of the $H F_{S C}$, i.e., $a_{\max }$ and $b_{\max }$, showed similar values within the different emotions, although both are subject dependent. The SCHF reveals a slight improvement in the reliability of sympathovagal balance estimation capable of discriminating neutral (relax) vs. positive (joy) valence, positive vs. negative (fear, sadness and anger) valences and negative (fear) vs. negative (sadness) valence. In accordance with our results, Goren Y. et al. [46] concluded the importance of redefining the boundary of the HF band for a correct evaluation of physiological changes of the ANS.

Mikuckas A. et al. [25] found that LF component and LF/HF ratio increased during exciting and sedative music, but decreased during silence. Moreover, Rantanen A. et al. [23] evidenced that negative valence elicitation, induced by unpleasant pictures, produced a higher $\mathrm{LF} / \mathrm{HF}$ ratio than neutral and pleasant pictures in a female cohort. Valenza G. et al. [47] investigated the synchronization between breathing patterns and heart rate during emotional visual elicitation by means of a set of neutral vs. increasing level of arousal images. In that study, it was found that the LF/HF ratio presented statistical differences between neutral and arousal sessions with higher $\mathrm{LF} / \mathrm{HF}$ ratio values while arousal sessions, in which sympathetic activity should be dominant. In our study, an increase in the $P_{L F n_{S C}}$ and $R_{S C}$ indices during joy (Fig. 6 (a-f)) was observed. Thus, joy could be associated with a sympathetic predominance. Additionally, $P_{L F n_{S C}}$ and $R_{S C}$ presented statistical differences discriminating neutral sessions vs. positive valence and J-F.

Besides the aforementioned elicitation types and emotions, population characteristics such as age could influence the results [46]. Thus, interpretation of the results should be addressed within this framework.

Although by means of the affect scales derived from the PANAS-X scale it was possible to differentiate between all emotion conditions, nor the indices derived from the SCHF methodology nor the other indices derived from the other HF band definitions were able to distinguish between all emotion conditions. This opens the door to explore other options as non-linear methodologies or a multimodal approach combining other physiological signals.

The newly introduced index $\rho_{\max }$, derived from the SCHF methodology, is a parameter suitable to be implemented on medical equipment, opening the door to help in identifying emotional behaviours in people suffering from mental pathologies. However, further studies are needed to test the validity and reliability of the proposed index outside laboratory settings.

\section{CONCLUSIONS}

In this study, human emotion recognition was assessed by HRV analysis. To increase the reliability of HRV measurements a novel methodology based on spectral correlation of HRV signal and respiration was proposed. Five emotional states corresponding to calm-neutral state (relax), positive valence (joy) and negative valences (fear, sadness and anger) were compared. The new proposed method, the Spectrum Correlation for High Frequency band, revealed an improvement in the reliability for sympathovagal balance estimation capable of discriminating between relax vs. joy, joy vs. each of the negative valences and fear vs. sadness. This method provided the novel index $\left(\rho_{\max }\right)$ which offers additional information for emotion recognition, based on the relationship between HRV and respiration.

\section{ACKNOWLEDGMENT}

Research supported by Ministerio de Economía y Competitividad (MINECO), FEDER; under the projects RTI2018097723-B-I00, DPI2016-75458-R and DPI2017-89827-R, by CIBER de Bioingeniería, Biomateriales y Nanomedicina through Instituto de Salud Carlos III, by Grupo Consolidado BSICoS from DGA (Aragón) and European Social Fund (EU). The computation was performed by the ICTS "NANBIOSIS", more specifically by the High Performance Computing Unit of the CIBER in Bioengineering, Biomaterials \& Nanomedicne (CIBERBBN).

\section{REFERENCES}

[1] G. Chanel, J.M. Kierkels, M. Soleymani, and T. Pun. Short-term emotion assessment in a recall paradigm. Int. J. Human-Computer Studies, 67(8):607-627, 2009.

[2] P.C. Petrantonakis and L.J. Hadjileontiadis. Emotion recognition from EEG using higher order crossings. IEEE Transactions on Information Technology in Biomedicine, 14(2):186-197, 2010.

[3] S. Koelstra, C. Mühl, M. Soleymani, J-S. Lee, A. Yazdani, T. Ebrahimi, T. Pun, A. Nijholt, and I. Patras. Deap: A database for emotion analysis using physiological signals. IEEE Transactions on Affective Computing, 3(1):18-31, 2012. 
[4] N. Kumar, K. Khaund, and S.M. Hazarika. Bispectral analysis of EEG for emotion recognition. Procedia Computer Science, 84:31-35, 2016.

[5] G. Mattavelli, M Rosanova, A. G. Casali, C. Papagno, and LJ Romero Lauro. Timing of emotion representation in right and left occipital region: Evidence from combined TMS-EEG. Brain and Cognition, 106:13-22, 2016.

[6] M. Soleymani, S. Asghari-Esfeden, Y. Fu, and M. Pantic. Analysis of EEG signals and facial expressions for continuous emotion detection. IEEE Transactions on Affective Computing, 7(1):17-28, 2016.

[7] M Postma-Nilsenov, E. Holt, L. Heyn, K. Groeneveld, and A. Finset. A case study of vocal features associated with galvanic skin response to stressors in a clinical interaction. Patient Education and Counseling, 99(8):1349-1354, 2016

[8] NN. Sudheesh and KP. Joseph. Investigation into the effects of music and meditation on galvanic skin response. ITBM-RBM, 21(3):158-163, 2000.

[9] K.H. Kim, S.W. Bang, and S.R. Kim. Emotion recognition system using short-term monitoring of physiological signals. Medical and Biological Engineering and Computing, 42(3):419-427, 2004.

[10] R. Bailón, L. Sörnmo, , and P. Laguna. A robust method for ECGbased estimation of the respiratory frequency during stress testing. IEEE Transactions on Biomedical Engineering, 53(7):1273-1285, 2006.

[11] D.S. Quintana, A.J. Guastella, T. Outhred, I.B. Hickie, and A.H. Kemp. Heart rate variability is associated with emotion recognition: direct evidence for a relationship between the autonomic nervous system and social cognition. Int. J. of Psychophysiol, 86(2):168-172, 2012.

[12] G. Valenza, M. Nardelli, A. Lanatá, C. Gentili, G. Bertschy, R. Paradiso, and E.P. Scilingo. Wearable monitoring for mood recognition in bipolar disorder based on history-dependent long-term heart rate variability analysis. IEEE Journal of Biomedical and Health Informatics, 18(5):1625$1635,2014$.

[13] A. Hernando, J. Lázaro, E. Gil, A. Arza, J. Mario, R. López-Antón, C. de la Cámara, P. Laguna, J. Aguiló, and R. Bailón. Inclusion of respiratory frequency information in heart rate variability analysis for stress assessment. IEEE Journal of Biomedical and Health Informatics, 20(4):1016-1025, 2016.

[14] B. Gómez and A. Escobar. La psiconeuroinmunología: bases de la relación entre los sistemas nervioso, endocrino e inmune. Facultad de Medicina de la Universidad Nacional Autónoma de México, 45(1), 2002.

[15] D.E. Mohrman and L.J. Heller. Cardiovascular Physiology. McGrawHill Interamericana, Mexico, 2006.

[16] F. Balada, C. Márquez, R. Nadal, D. Redolar, and J. Silvestre. Farmacología y endocrinología del comportamiento. Editorial UOC, Spain, 2012.

[17] A. Arza, J.M. Garzón-Rey, J. Lázaro, E. Gil, R. López-Antón, C. de la Cámara, P. Laguna, R. Bailón, and J. Aguiló. Measuring acute stress response through physiological signals: Towards a quantitative assessment of stress. Medical \& Biological Engineering \& Computing, pages $1-17,2018$

[18] M.J. Turlough Fitzgerald, G. Gruener, and E. Mtui. Clinical neuroanatomy and neuroscience. Elsevier Saunders, España, 2012.

[19] A. Despopoulos and S. Silbernagl. Color Atlas of Physiology. Basic Sciences (Thieme), USA, 2003

[20] H. Cohen, J. Benjamin, A. B. Geva, M. A. Matar, Z. Kaplan, and M. Kotler. Autonomic dysregulation in panic disorder and in posttraumatic stress disorder: application of power spectrum analysis of heart rate variability at rest and in response to recollection of trauma or panic attacks. Psychiatry Research, 96(1):1-13, 2000.

[21] H. A. Demaree and D. E. Everhart. Healthy high-hostiles: reduced parasympathetic activity and decreased sympathovagal flexibility during negative emotional processing. Personality and Individual Differences, $36: 457469,2004$

[22] X. Bornasa, J. Llabrés, M. Noguerac, A. M. López, F. Barceló, M. Tortella-Feliu, and M. A. Fullana. Looking at the heart of low and high heart rate variability fearful flyers: self-reported anxiety when confronting feared stimuli. Biological Psychology, 70(3):182-187, 2005.

[23] A. Rantanen, S.J. Laukka, M. Lehtihalmes, and T. Seppaänen. Heart rate variability reflecting from oral reports on negative experience. Procedia Soc. Behav. Sci., 5:483-487, 2010.

[24] F. C. M. Geisler, N. Vennewald, T. Kubiak, and H. Weber. The impact of heart rate variability on subjective well-being is mediated by emotion regulation. Personality and Individual Differences, 49:723728, 2010.

[25] A. Mikuckas, I. Mikuckiene, A. Venckauskas, E. Kazanavicius, R. Lukas, and I. Plauska. Emotion recognition in human computer interaction systems. Elektronika ir Elektrotechnika, 20(10):1392-1215, 2014.
[26] Task Force of ESC and NASPE. Heart rate variability. standards of measurement, physiological interpretation, and clinical use. Eur. Heart J., 17:354-381, 1996

[27] F. Yasuma and J.I. Hayano. Respiratory sinus arrhythmia: Why does the heartbeat synchronize with respiratory rhythm? Chest, 125(2):683-690, 2004.

[28] R. Bailón, P. Laguna, L. Mainardi, and L. Sörnmo. Analysis of heart rate variability using time-varying frequency bands based on respiratory frequency. In IEEE EMBS International Conference on Engineering in Medicine and Biology Society, 29th International Conference on, pages 6674-6677, 2007.

[29] R. Bailón, L. Mainardi, M. Orini, L. Sörnmo, and P. Laguna. Analysis of heart rate variability during stress testing using respiratory information. Biomed. Signal Process. Control, 5(4):299-310, 2010.

[30] M. Turon, S. Fernandez-Gonzalo, M. Jodar, G. Goma, J. Montanya, D. Hernando, R. Bailón, C. de Haro, V. Gomez-Simon, J. Lopez-Aguilar, R. Magrans, M. Martinez-Perez, C. Oliva, and L. Blanch. Feasibility and safety of virtual-reality-based early neurocognitive stimulation in critically ill patients. Annals of Intensive Care, 7(81):1-11, 2017.

[31] M. Daoud, P. Ravier, and O. Buttelli. Use of cardiorespiratory coherence to separate spectral bands of the heart rate variability. Biomedical Signal Processing and Control, 46:260-267, 2018.

[32] S. Kontaxis, M. Orini, E. Gil, M. Posadas-De Miguel, M.L. Bernal, J. Aguiló, C. de la Camara, P. Laguna, and R. Bailón. Heart rate variability analysis guided by respiration in major depression disorder. Proceedings of the XLV International Conference on Computing in Cardiology, 7(81):1-4, 2018.

[33] M. Orini, R. Bailón, L.T. Mainardi, P. Laguna, and P. Flandrin. Characterization of dynamic interactions between cardiovascular signals by time-frequency coherence. IEEE Transactions on Biomedical Engineering, 59(3):663-673, 2012.

[34] M. Orini, R. Bailón, P. Laguna, L.T. Mainardi, and R. Barbieri. A multivariate time-frequency method to characterize the influence of respiration over heart period and arterial pressure. EURASIP Journal on Advances in Signal Processing, 2012(214):1-17, 2012.

[35] D. Watson and L.A. Clark. The PANAS-X: Manual for the Positive and Negative Affect Schedule - Expanded Form. Department of Psychological \& Brain Sciences Publications, University of Iowa, 1999.

[36] J. P. Martínez, R. Almeida, S. Olmos, A. P. Rocha, and P. Laguna Wavelet-based ECG delineator: evaluation on standard databases. IEEE Transactions on Biomedical Engineering, 51:570-581, 2004.

[37] J. Mateo and P. Laguna. Analysis of heart rate variability in the presence of ectopic beats using the heart timing signal. IEEE Transactions on Biomedical Engineering, 50(3):334-343, 2003.

[38] R. Bailón, G. Laouini, C. Grao, M. Orini, and P. Laguna. The integral pulse frequency modulation model with time-varying threshold: Application to heart rate variability analysis during exercise stress testing. IEEE Transactions on Biomedical Engineering, 58(3):642-652, 2011.

[39] M.T. Valderas, J. Bolea, P. Laguna, M. Vallverdú, and R. Bailón. Human emotion recognition using heart rate variability analysis with spectral bands based on respiration. In IEEE EMBS International Conference on Engineering in Medicine and Biology Society, 37th International Conference on, pages 6674-6677, 2015.

[40] M. Orini, R. Bailón, L.T. Mainardi, and P. Laguna. Synthesis of HRV signals characterized by predetermined time-frequency structure by means of time-varying arma models. Biomedical Signal Processing and Control, 7:141-150, 2012.

[41] J. Bolea, E. Pueyo, P. Laguna, and R. Bailón. Non-linear HRV indices under autonomic nervous system blockade. In 36nd Annual International Conference of the IEEE EMBS, pages 3252-3255, 2014.

[42] W.R. Rice. Analyzing tables of statistical test. Biomedical Signal Processing and Control, 43(1):223-225, 1989.

[43] H. Ney, S. Martin, and F. Wessel. Statistical language modeling using leaving-one-out. In S. Young and G. Bloothooft, editors, CorpusBased Methods in Language and Speech Processing. Text, Speech and Language Technology, chapter 6, pages 174-207. Springer, Dordrecht, 1997.

[44] P Ekman. An argument for basic emotions. Cognition and Emotion, 6 (3/4):169-200, 1969.

[45] R.E. Jack, O.G. Garrod, and P.G. Schyns. Dynamic facial expressions of emotion transmit an evolving hierarchy of signals over time. Current biology, 24(2):187-92, 2014.

[46] Y. Goren, L.R. Davrath, I. Pinhas, E. Toledo, and S. Akselrod. Individual time-dependent spectral boundaries for improved accuracy in timefrequency analysis of heart rate variability. IEEE Trans. Biomed. Eng., 53(1):35-42, 2006. 
[47] G. Valenza, A. Lanatá, and E.P. Scilingo. Oscillations of heart rate and respiration synchronize during affective visual stimulation. IEEE Transactions on Information Technology in Biomedicine, 16(4):683-690, 2012.

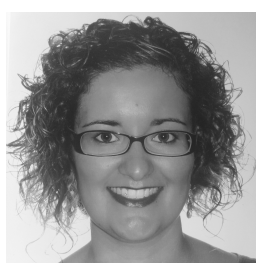

María Teresa Valderas was born in Barcelona in 1983. She received the degree in Physics in 2007. She obtained a joint M.Sc. in Biomedical Engineering from the University of Barcelona and Barcelona Tech, Spain in 2009. She did her master thesis at the University of Applied Science of Jena (Germany, 6 months, 2009) about extraction of the respiratory signal from the electrocardiogram. She was developing and characterising macroporous hydroxiapatite scaffolds with proteins for bone regeneration in Biomedical Engineering Research Center, Spain (2009-2012). She was project manager in the field of biomedical engineering innovative projects (2012-2014). In 2017 she obtained a postgraduate in psychoneuroimmunoendocrinology by the University of Barcelona. Currently she is involved in the Ph.D in the area of Biomedical Engineering, both at the University of Zaragoza and in the Barcelona Tech. Her research is focused on human emotion recognition by linear and non-linear indices.

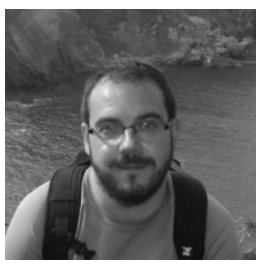

Juan Bolea Juan Bolea was born in Zaragoza in 1981. He received the M. Sc. degree in Physics in 2006 and the $\mathrm{PhD}$ in Biomedical Engineering in 2018. He worked as a software developer supporting BSICoS group gathering advanced signal processing tools in a user-friendly signal processing platform named BioSigBrowser. His research activities are mainly focused on nonlinear techniques to assess heart rate variability.

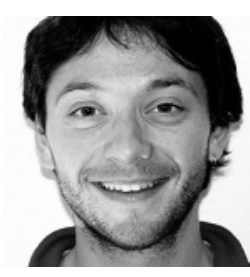

Michele Orini is a Research Fellow at the Institute of Cardiovascular Science, University College of London, UK. His work involves studying the electrical activity of the heart and some of its dysfunctions related to sudden cardiac death. He obtained a joint Ph.D. in Biomedical Engineering from the University of Zaragoza, Spain, and the Politecnico di Milano, Italy. His Ph.D. was in the field of non-stationary biomedical signal processing and focused on the dynamic interactions between cardiovascular signals, such as heart rate, arterial pressure, respiration, photopletismographic signal, etc. He received the M.Sc. in Biomedical Engineering from the Politecnico di Milano, Italy, and a degree in engineering from the Ecole Centrale Paris, France in 2006. He also worked at the Barcelona Tech (Spain, 6 months, 2008), Ecole Normale de Lyon (France, 4 months, 2010) and at Harvard Medical School of Boston (US, 4 months, 2011) as a visiting student.

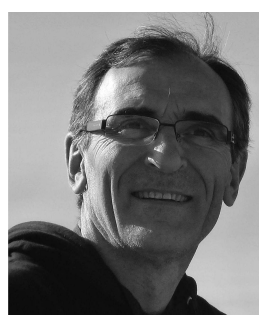

Pablo Laguna is Full Professor of Signal Processing and Communications in the Department of Electrical Engineering at the Engineering School, where he has being vice-dean for international relation (19992002), and a researcher at the Aragón Institute for Engineering Research (I3A), both at University of Zaragoza, Spain, where he has being responsible of the Biomedical Engineering division of the I3A (2000-2011) and of the master in Biomedical Engineering (2003-2010). He is also member, and has served as scientific director (2011-2015), of the Spanish Center for Biomedical Engineering, Biomaterial and Nano-medicine Research CIBER-BBN. His professional research interests are in Signal Processing, in particular applied to Biomedical applications. He has co-authored more than 120 research papers on this topic, over 250 international conference papers, and has advise $12 \mathrm{Ph} . \mathrm{D}$ Thesis. He has lead a broad number of projects on biomedical signal interpretation especially in the cardiovascular domain, most of them with international collaborations at clinical and engineering sites. $\mathrm{He}$ is having some international scientific responsibilities, as serving as pastpresident of the board of directors of Computing in Cardiology conference, editor of the digital signal processing journal (Eurasip), and of the Medical and Biological Engineering and Computing, organizer of different scientific conferences, etc. He is also responsible of the Ph.D. program in Biomedical Engineering at Zaragoza University. He is Fellow of the IEEE. He is, together with L. Sörnmo, the author of Bioelectrical Signal Processing in Cardiac and Neurological Applications, book (Elsevier, 2005).

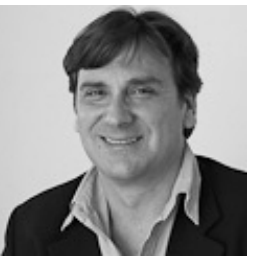

Carlos Orrite received in 1989 the master degree in Industrial Engineering and in 1994 the master degree in Biomedical Engineering at the Zaragoza University, working in the field of medical instrumentation. In 1997 he did his Ph.D on Computer Vision at the Zaragoza University, winner of the 1997 Zaragoza University Thesis Prize in Engineering. He is currently associate professor at the Department of Electronics and Communications Engineering, at the University of Zaragoza. Since January 2013 he is accredited as Full Professor by the National Agency for Quality Assessment and Accreditation (ANECA). His research interests are in the area of computer vision, biometrics, human motion analysis, wearable computing and Augmented Reality. Since 2005 up to date he has been leading the Computer Vision Group at the University of Zaragoza. From October of 2011 to November of 2015 he has been the Associate Director of I3A, responsible of the Information and Communication Technologies Division. Since 2012 up to date he is the secretary of the Spanish Association of Pattern Recognition and Image Analysis (AERFAI).

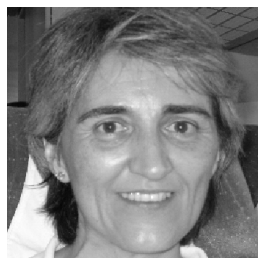

Montserrat Vallverdú is senior researcher at Biomedical Engineering Research Centre (CREB) and Associate Professor at Barcelona Tech (UPC), specialization in automatic control engineering at the Graduate and Masters levels and biomedical signal processing and modeling at $\mathrm{PhD}$ level. She is also member of the Bioinformatics and Biomedical Signals Laboratory (B2SLab) research group at the UPC and CIBER of Bioengineering, Biomaterials and Nanomedicine (CIBER-BBN). Her main research focuses on the complexity and time-frequency analysis to the recovery of the clinically useful hidden information in cardiac, respiratory and brain signals, and on the identification and simulation of their involved physiological control mechanisms.

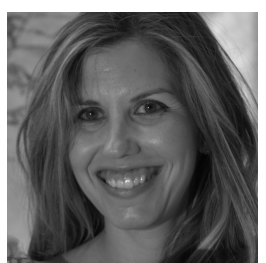

Raquel Bailón was born in Zaragoza, Spain, in 1978. She received the M.S. degree in Telecommunication Engineering and the Ph.D. degree in Biomedical Engineering from the University of Zaragoza (UZ), Zaragoza, Spain, in 2001 and 2006, respectively. In 2003, she was an Assistant Professor in the Department of Electronic Engineering and Communications, UZ, where she is currently an Associate Professor. She is also a Researcher with the Aragon Institute for Engineering Research, UZ, and also with the Centro de Investigación Biomédica en Red en Bioingeniería, Biomateriales y Nanomedicina, Spain. Her current research interests include the biomedical signal processing field, especially in the analysis of the dynamics and interactions of cardiovascular signals. 